Electronic version of an article published as [Lecture Notes in Computer Science, 2007, No. 4818, p. 536-544] [DOI: http://dx.doi.org/10.1007/978-3-540-78827-0_61]

(C) [copyright Springer Verlag] 


\title{
Semi-Dynamic demand in a Non-Permutation Flowshop with Constrained Resequencing Buffers ${ }^{\star}$
}

\author{
Gerrit Färber $^{1}$, Said Salhi ${ }^{2}$, and Anna M. Coves Moreno ${ }^{1}$ \\ ${ }^{1}$ Institut d'Organització i Control de Sistemes Industrials, \\ Universitat Politècnica de Catalunya, Barcelona, Spain \\ 2 The Center for Heuristic Optimisation, Kent Business School, \\ University of Kent, Canterbury, UK \\ Gerrit_Faerber@gmx.de; s.salhi@kent.ac.uk; \\ Anna.Maria.Coves@upc.edu
}

\begin{abstract}
This work presents the performance comparison of two conceptually different approaches for a mixed model non-permutation flowshop production line. The demand is a semi-dynamic demand with a fixed job sequence for the first station. Resequencing is permitted where stations have access to intermediate or centralized resequencing buffers. The access to the buffers is restricted by the number of available buffer places and the physical size of the products. An exact approach, using Constraint Logic Programming (CLP), and a heuristic approach, a Genetic Algorithm (GA), were applied.
\end{abstract}

Keywords: Semi-dynamic demand, Constraint Logic Programming, Genetic Algorithm, Non-Permutation Flowshop, Mixed model assembly line

\section{Introduction}

Mixed model production lines consider more than one model being processed on the same production line in an arbitrary sequence. Nevertheless, the majority of publications are limited to solutions which determine the job sequence before the jobs enter the line and maintain it without interchanging jobs until the end of the production line, known as permutation flowshop. In the case of more than three stations and with the objective function to minimize the makespan, a unique permutation for all stations is no longer optimal. In [1] and [2] studies of the benefits of using non-permutation flowshops are presented.

Various designs of production lines, which permit resequencing of jobs, exist [3], [4], [5] and [6]. Resequencing of jobs on the line is even more relevant with the existence of an additional cost or time, occurring when at a station the succeeding job is of another model, known as setup-cost and setup-time [7].

* This work is partially supported by the Ministry of Science and Technology, and the funding for regional research DPI2004-03472 
The case of infinite buffers is basically a theoretical case in which no limitation exists with respect to the number of jobs that may be buffered between two stations. Surveys on heuristics treating the case of infinite buffers are presented in [8] and [9]. Approaches which consider a limited number of buffer places for the flowshop problem are studied in [10], [11], [12], [13], [14], [15] and [16]. In [17] and [18] limited resequencing possibilities are considered for jobshop problems.

The introduction of resequencing possibilities generally leads to additional costs, caused by additional equipment to be mounted, like buffers, but also extra efforts in terms of logistics complexity may arise. In the case in which there exist jobs with large and small physical size, the investment for additional resequencing equipment can be reduced by, e.g., only giving small jobs the possibility to resequence. Consequently, only small resequencing buffer places are installed. Following this concept, in a chemical production line where the demand of customers is different, only resequencing tanks that permit to resequence the request of a small customer order are used.

In what follows, the problem is formulated in more detail, and the exact and the heuristic approaches are explained. Thereafter promising results are presented for medium and large sized problems, which demonstrate the relevance of the proposed concept, followed by the conclusions.

\section{Problem definition}

This paper considers a mixed model non-permutation flowshop with the possibility of resequencing jobs between consecutive stations. The jobs $\left(J_{1}, J_{2}, \ldots, J_{j}\right.$, $\left.\ldots, J_{n}\right)$ pass consecutively through the stations $\left(I_{1}, I_{2}, \ldots, I_{i}, \ldots, I_{m}\right)$ and after determined stations, off-line buffers $B_{i}$ permit to resequence jobs. The buffer provides various buffer places $\left(B_{i, 1}, B_{i, 2}, \ldots\right)$ and each buffer place is restricted

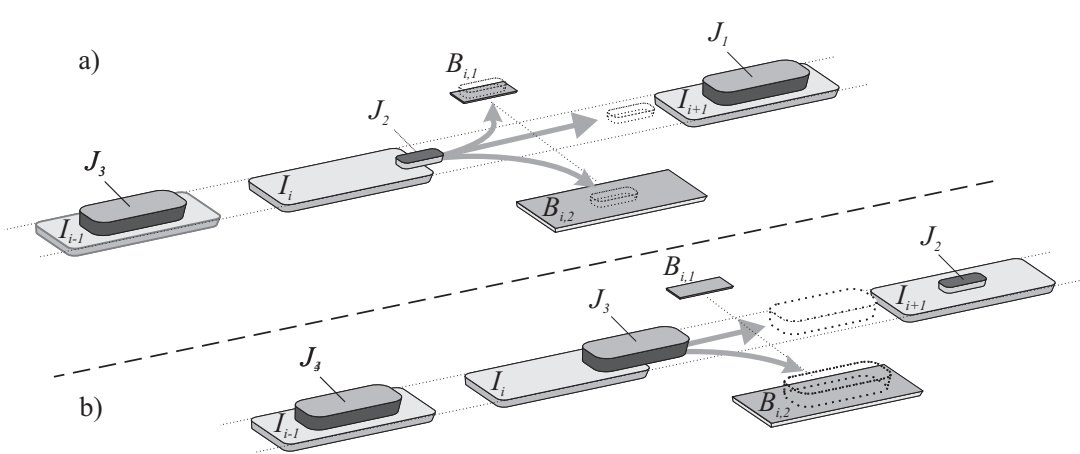

Fig. 1: Scheme of the considered flowshop. The jobs $J_{j}$ pass consecutively through the stations $I_{i}$. The buffer $B_{i}$ permits to temporally store a job with the objective of reinserting it at a later position in the sequence. a) Job $J_{2}$ can pass through any of the two buffer places $B_{i, 1}$ or $B_{i, 2}$ of buffer $B_{i}$. b) Job $J_{3}$ can pass only through buffer place $B_{i, 2}$, due to its physical size. 
by the physical size of the jobs to be stored. As can be seen in figure 1a, job $J_{2}$ can be stored in buffer place $B_{i, 1}$ as well as in $B_{i, 2}$. Whereas, the next job $J_{3}$ can be stored only in buffer place $B_{i, 2}$, because of the physical size of the job exceeding the physical size of buffer place $B_{i, 1}$, see figure $1 \mathrm{~b}$.

The buffers are located off-line, in a first step accessible from a single station (intermediate case). Then, for an additional benefit, a single resequencing buffer is used, accessible from various stations (centralized case). The objective function is the weighted sum of the makespan and the setup-cost, where there is no weight associated with the setup-time though this is indirectly included in the calculation of the makespan. An exact approach, using Constraint Logic Programming (CLP) and a heuristic approach, using a Genetic Algorithm (GA), were applied to the problem under study.

\section{Approaches}

\subsection{Exact approach: CLP}

The concept of CLP can be described as a powerful extension of conventional logic programming [19]. It involves the incorporation of constraint languages and constraint solving methods into logic programming languages [20].

The formulation used here is explained in more detail in [21] and was implemented in OPL Studio version 3.7. Apart from job and station precedences, the CLP formulation determines the jobs which are to be taken off the line for the purpose of resequencing, given that a free buffer place is available and that the physical size of the job does not exceed the physical size of the buffer place. The formulation also includes computational enhancements like imposing the start time of jobs and the reduction of the size of the variables and considers the intermediate as well as the centralized location of the resequencing buffers and includes

\subsection{Heuristic approach: GA}

The concept of Genetic Algorithms (GA) can be understood as the application of the principles of evolutionary biology, also known as the survival of the fittest [22], [23]. Genetic algorithms are typically implemented as a computer simulation in which a population of chromosomes, each of which represents a solution of the optimization problem, evolves toward better solutions. The evolution starts from an initial population which may be determined randomly. In each generation, the fitness of the whole population is evaluated and multiple individuals are stochastically selected from the current population, based on their fitness and modified to form a new population. The alterations are biologically-derived techniques, commonly achieved by inheritance, mutation and crossover. Multiple Genetic Algorithms were designed for mixed model assembly lines such as [24], [25], [26] and [27]. 
The heuristic used here is a variation of the GA explained in [28]. The genes represent the jobs which are to be sequenced. The chromosomes $v$, determined by a series of genes, represent a sequence of jobs. A generation is formed by $R$ chromosomes and the total number of generations is $G$. In the permutation case, the size of a chromosome is determined by the number of jobs, the fraction $\Pi$. In the non-permutation case, the chromosomes are $L+1$ times larger, resulting in the fractions $\Pi_{1}^{\prime}, \ldots, \Pi_{L+1}^{\prime}$, being $L$ the number of resequencing possibilities. In both cases, special attention is required when forming the chromosomes, because of the fact that for each part of the production line every job has to be sequenced exactly one time.

The relevant information for each chromosome is its fitness value (objective function), the number of job changes and the indicator specifying if the chromosome represents a feasible solution. A chromosome is marked infeasible and is imposed with a penalty. This situation arises if a job has to be taken off the line and no free resequencing buffer place is available or the physical size of the job exceeds the size limitation of the available resequencing buffer places. When two solutions result in the same fitness, the one with fewer job changes is preferred. In [29] the detailed formulation can be found.

\section{Performance Study}

The performance study considers a medium sized problem with 10 stations and up to 10 jobs. This is applied to the exact (CLP) as well as to the heuristic approach (GA). The second instance uses a large problem with 5 stations and up to 100 jobs where the heuristic approach (GA) is applied only.

\subsection{Instance-1: Medium sized problem (CLP versus GA)}

A flowshop which consists of 10 stations is considered. After station 3,5 and 8 a single intermediate buffer place is located. The range of the production time is $[1 \ldots 100]$, for the setup cost $[2 \ldots 8]$ and for the setup time [1...5]. The number of jobs is varied in the range of 4 to 10 and the objective function is the weighted sum of the makespan (factor 1.0) and the setup cost (factor 0.3), where the setup time is not concerned with a weight but is indirectly included in the calculation of the makespan.

Three differently sized buffer places (large, medium, small) are available and the ratio of jobs is $\frac{3}{10}, \frac{3}{10}$ and $\frac{4}{10}$ for large, medium and small, respectively. The allocation of the buffer places to the buffers considers five scenarios for the intermediate case ("I111", "I231", "I132", "I222", "I333") and three scenarios for the centralized case ("C1", "C2", "C3"). "I132" represents 1 small, 1 large and 1 medium buffer place, located as intermediate resequencing buffer places after stations 3, 5 and 8, respectively. "C2" represents 1 medium buffer place, located as a centralized buffer place, accessible from stations 3, 5 and 8. "I333" and "C3" are the two cases which provide the largest flexibility in terms of physical size restrictions. 


\begin{tabular}{|ccccc|}
\hline$I 111$ & $\mid 231$ & $\mid 132$ & $\mid 222$ & $\mid 333$ \\
\hline 480,9 & $\mathbf{4 8 0 , 7}$ & $\mathbf{4 8 0 , 7}$ & 480,9 & $\mathbf{4 8 0 , 7}$ \\
$\mathbf{4 9 0 , 7}$ & $\mathbf{4 9 0 , 7}$ & $\mathbf{4 9 0 , 7}$ & $\mathbf{4 9 0 , 7}$ & $\mathbf{4 9 0 , 7}$ \\
627,0 & $\mathbf{6 2 0 , 1}$ & $\mathbf{6 2 0 , 1}$ & $\mathbf{6 2 0 , 1}$ & $\mathbf{6 2 0 , 1}$ \\
627,7 & 627,7 & 625,0 & 625,0 & $\mathbf{6 0 9 , 5}$ \\
646,6 & 646,6 & 644,8 & 644,8 & 644,8 \\
719,4 & 719,4 & 716,1 & 716,1 & 716,1 \\
$\mathbf{7 8 6 , 5}$ & 763,2 & $\mathbf{7 6 2 , 5}$ & 785,9 & $\mathbf{7 9 1 , 2}$ \\
\hline
\end{tabular}

\begin{tabular}{|ccc|}
\hline C1 & C2 & C3 \\
\hline 480,9 & $\mathbf{4 8 0 , 7}$ & $\mathbf{4 8 0 , 7}$ \\
$\mathbf{4 9 0 , 7}$ & $\mathbf{4 9 0 , 7}$ & $\mathbf{4 9 0 , 7}$ \\
628,5 & 622,2 & 622,2 \\
628,0 & 627,7 & 616,1 \\
646,6 & 644,8 & $\mathbf{6 3 2 , 3}$ \\
$\mathbf{7 1 9 , 4}$ & 716,1 & $\mathbf{7 1 2 , 1}$ \\
$\mathbf{7 8 6 , 5}$ & 786,5 & 764,0 \\
\hline
\end{tabular}

Table 1: Semi-dynamic demand using the exact approach (CLP).

The results of the CLP are shown in table 1. In all cases, when offline resequencing buffers are considered, the results are improved compared to the permutation sequence. In the studied flowshop, an average of $4.3 \%$ is achieved for the CLP, whereas, in the case of the GA, see table 2, the average is $3.7 \%$.

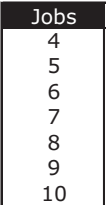

\begin{tabular}{|l|}
\hline Perm \\
\hline 483,1 \\
552,0 \\
647,5 \\
636,5 \\
673,6 \\
744,3 \\
8137 \\
\hline
\end{tabular}

\begin{tabular}{|ccccc|}
\hline$I 111$ & $\mid 231$ & $\mid 132$ & $\mid 222$ & $\mid 333$ \\
\hline 480,9 & $\mathbf{4 8 0 , 7}$ & $\mathbf{4 8 0 , 7}$ & $\mathbf{4 8 0 , 7}$ & $\mathbf{4 8 0 , 7}$ \\
$\mathbf{4 9 0 , 7}$ & $\mathbf{4 9 0 , 7}$ & $\mathbf{4 9 0 , 7}$ & $\mathbf{4 9 0 , 7}$ & $\mathbf{4 9 0 , 7}$ \\
627,0 & $\mathbf{6 2 0 , 1}$ & $\mathbf{6 2 0 , 1}$ & $\mathbf{6 2 0 , 1}$ & $\mathbf{6 2 0 , 1}$ \\
$\mathbf{6 2 7 , 7}$ & 628,8 & 626,1 & 625,0 & $\mathbf{6 0 9 , 5}$ \\
669,2 & 651,3 & 646,0 & 647,2 & 638,2 \\
736,5 & 724,2 & 728,6 & 721,8 & $\mathbf{7 0 9 , 4}$ \\
808,3 & 788,0 & 781,7 & 805,1 & $\mathbf{7 5 7 , 5}$ \\
\hline
\end{tabular}

\begin{tabular}{ccc} 
C1 & C2 & C3 \\
480,9 & $\mathbf{4 8 0 , 7}$ & $\mathbf{4 8 0 , 7}$ \\
$\mathbf{4 9 0 , 7}$ & $\mathbf{4 9 0 , 7}$ & $\mathbf{4 9 0 , 7}$ \\
628,5 & 622,7 & 622,2 \\
629,1 & 628,5 & 616,3 \\
672,4 & 653,4 & $\mathbf{6 3 7 , 0}$ \\
736,5 & 729,5 & 714,7 \\
809,2 & 805,9 & 772,2 \\
\hline
\end{tabular}

Table 2: Semi-dynamic demand using the heuristic approach (GA).

In the case of the exact approach, as well as in the GA, the semi-dynamic demand with a fixed job sequence for the first station, leads to a considerable improvement. In table 3 the improvement of the CLP with respect to the GA is shown. For up to 5 jobs, both methods achieve the same solutions. When 6 or more jobs are to be sequenced, in general, the CLP outperforms the GA when smaller buffer places are used.

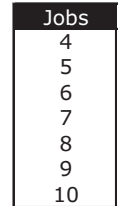

\begin{tabular}{|l||}
\hline Perm \\
\hline 483,1 \\
552,0 \\
647,5 \\
636,5 \\
673,6 \\
744,3 \\
813,7 \\
\hline
\end{tabular}

\begin{tabular}{|lllll|}
\hline $\mid 111$ & $\mid 231$ & $\mid 132$ & $\mid \mathbf{I 2 2 2}$ & $\mid \mathbf{3 3 3}$ \\
\hline $\mathbf{0 , 0} \%$ & $\mathbf{0 , 0} \%$ & $\mathbf{0 , 0} \%$ & $\mathbf{0 , 0} \%$ & $\mathbf{0 , 0} \%$ \\
$\mathbf{0 , 0} \%$ & $\mathbf{0 , 0} \%$ & $\mathbf{0 , 0} \%$ & $\mathbf{0 , 0} \%$ & $\mathbf{0 , 0} \%$ \\
$\mathbf{0 , 0} \%$ & $\mathbf{0 , 0} \%$ & $\mathbf{0 , 0} \%$ & $\mathbf{0 , 0} \%$ & $\mathbf{0 , 0} \%$ \\
$\mathbf{0 , 0} \%$ & $0,2 \%$ & $0,2 \%$ & $\mathbf{0 , 0} \%$ & $\mathbf{0 , 0} \%$ \\
$3,4 \%$ & $0,7 \%$ & $0,2 \%$ & $0,4 \%$ & $-1,0 \%$ \\
$2,3 \%$ & $0,7 \%$ & $1,7 \%$ & $0,8 \%$ & $-0,9 \%$ \\
$2,7 \%$ & $3,1 \%$ & $2,5 \%$ & $2,4 \%$ & $-4,5 \%$ \\
\hline
\end{tabular}

\begin{tabular}{ccc}
$\mathrm{C} 1$ & $\mathrm{C} 2$ & $\mathrm{C} 3$ \\
$\mathbf{0 , 0} \%$ & $\mathbf{0 , 0} \%$ & $\mathbf{0 , 0} \%$ \\
$\mathbf{0 , 0} \%$ & $\mathbf{0 , 0} \%$ & $\mathbf{0 , 0} \%$ \\
$\mathbf{0 , 0} \%$ & $0,1 \%$ & $\mathbf{0 , 0} \%$ \\
$0,2 \%$ & $0,1 \%$ & $\mathbf{0 , 0} \%$ \\
$3,8 \%$ & $1,3 \%$ & $0,7 \%$ \\
$2,3 \%$ & $1,8 \%$ & $0,4 \%$ \\
$2,8 \%$ & $2,4 \%$ & $1,1 \%$ \\
\hline
\end{tabular}

Table 3: Comparison of the GA and the Constrained Logic Programming for semidynamic demand. The values show the improvement of the CLP with respect to the GA.

The execution time in the vast majority of the cases was inferior to 600 seconds for the case of the CLP. The execution time of the GA, limited to 1000 iterations, required up to 55 seconds; increasing the number of iterations did not result in a major improvement. In general, the solutions of the CLP show better results in the more restricted problem. Nevertheless, the GA has to process a large number of infeasible solutions when the resequencing possibilities are heavily restricted. The GA consequently performs better in the less restricted the problem. This behavior was also observed when the demand is not a semidynamic demand, considering that the jobs can be resequenced before they enter the production line. 


\subsection{Instance-2: Large problem (GA)}

A flowshop which consists of 5 stations is considered. The range of the production time is $[0 \ldots 20]$ such that for some jobs zero-processing time at some stations exists, for the setup cost $[2 \ldots 8]$ and for the setup time [1...5]. The objective function is the weighted sum of the makespan (factor of 1.0) and the setup cost (factor of 0.3). The setup time has no weight associated with but is indirectly included in the calculation of the makespan. For the intermediate case, three scenarios are considered: in I22, both buffers are provided with two buffer places each; in I20, only station 2 has access to a resequencing buffer with two places; and in I02, only station 3 has access to a resequencing buffer with two places. For the centralized case, station 2 and station 3 have access to a centralized buffer with two places (C2), three places (C3) and four places (C4).

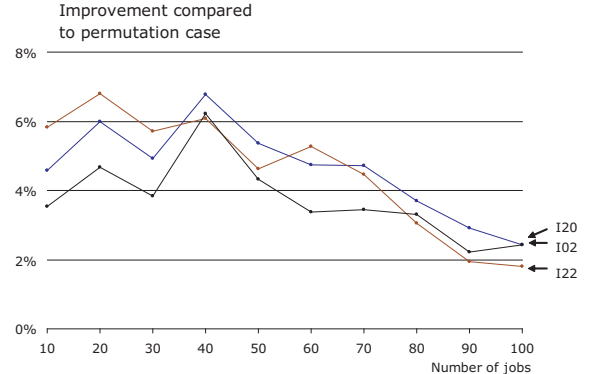

a) Intermediate case

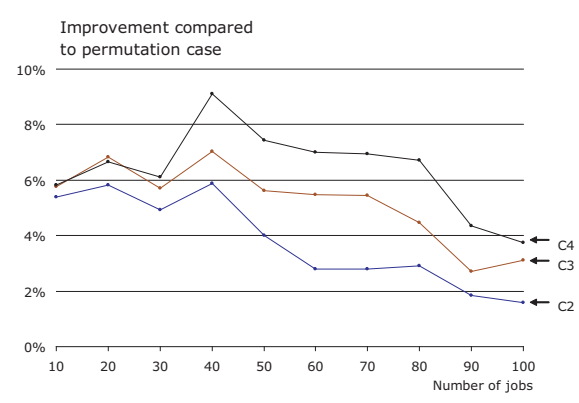

b) Centralized case

Fig. 2: Semi-dynamic demand without sequencing before the first station: a) In I22, both buffers are provided with two buffer places each; in I20, only station 2 has access to a resequencing buffer with two places; and in I02, only station 3 has access to a resequencing buffer with two places. b) Station 2 and station 3 have access to a centralized buffer with two buffer places (C2), three buffer places (C3) and four buffer places (C4)

Figure 2 shows the amounts of improvement which are achieved by the GA, when resequencing of a randomly generated sequence within the production line (semi-dynamic case) is permitted, compared to the case without resequencing. In the intermediate case, see figure $2 \mathrm{a}$, the use of the two resequencing possibilities (I22) achieves best results up to 30 jobs, then, the case of one resequencing possibility at station 2 (I20) outperforms in nearly all cases until 100 jobs. Even though I22 provides more flexibility in terms of resequencing, the GA performs better for instances with fewer resequencing possibilities when more than 30 jobs are to be sequenced. This comes from the fact that the length of the chromosomes is dependent on the number of resequencing possibilities $(L)$.

In the centralized case, see figure $2 \mathrm{~b}$, the variable parameter is the number of buffer places. The use of two buffer places (C2) in all of the considered cases is inferior compared to the case of three and four buffer places $(\mathrm{C} 3, \mathrm{C} 4)$. Until 30 jobs, the cases $\mathrm{C} 3$ and $\mathrm{C} 4$ achive nearly equivalent results which means that the fourth buffer place is not required. Then, for 40 jobs and more, the fourth buffer place shows a considerable impact on the possible improvements, compared to the case without resequencing. 


\section{Conclusions}

This paper has presented the performance comparison of two conceptually different approaches for a mixed model non-permutation flowshop production line. The demand is a semi-dynamic demand with a fixed job sequence for the first station and resequencing is permitted where stations have access to intermediate or centralized resequencing buffers. Furthermore, the access to the buffers is restricted by the number of available buffer places and the physical size of the products.

The accomplished performance study demonstrated the effectiveness of resequencing jobs within the line. The exact approach, using Constraint Logic Programming (CLP), outperforms the Genetic Algorithm (GA), when the physical size of the resequencing buffer places is limited. Due to the limited applicability of the exact approach, the performance study for larger problems of up to 100 jobs was performed using the GA. The chromosome size is dependent on the number of resequencing possibilities and therefore the performance of the GA for more than 30 jobs shows better results when fewer resequencing possibilities are present.

The results revealed the benefits that come with a centralized buffer location, compared to the intermediate buffer location. It either improves the solution or leads to the use of fewer resequencing buffer places. An increased number of large buffer places clearly improves the objective function and including buffers, constrained by the physical size of jobs to be stored, on one side limits the solutions but on the other side minimizes the necessary buffer area.

In order to take full advantage of the possibilities of resequencing jobs in a mixed model flowshop, additional installations may be necessary to mount, like buffers, but also extra efforts in terms of logistics complexity may arise. The additional effort is reasonable if it pays off the necessary investment.

\section{References}

[1] Potts, C., Shmoys, D., Williamson, D.: Permutation vs. non-permutation flow shop schedules. Operations Research Letters 10(5) (1991) 281-284

[2] Liao, C., Liao, L., Tseng, C.: A performance evaluation of permutation vs. nonpermutation schedules in a flowshop. International Journal of Production Research 44(20) (2006) 4297-4309

[3] Lee, H., Schaefer, S.: Sequencing methods for automated storage and retrieval systems with dedicated storage. Computers and Industrial Engineering 32(2) (1997) 351-362

[4] Lahmar, M., Ergan, H., Benjaafar, S.: Resequencing and feature assignment on an automated assembly line. IEEE Transactions on Robotics and Automation 19(1) (2003) 89-102

[5] Engstrőm, T., Jonsson, D., Johansson, B.: Alternatives to line assembly: Some swedish examples. International Journal of Industrial Ergonomics 17(3) (1996) $235-245$

[6] Rachakonda, P., Nagane, S.: Simulation study of paint batching problem in automobile industry. http://sweb.uky.edy/ pkrach0/Projects/MFS605Project.pdf (2000) consulted 14.07.2004.

[7] Bolat, A.: Sequencing jobs on an automobile assembly line: objectives and procedures. International Journal of Production Research 32(5) (1994) 1219-1236 
[8] Liesegang, G., Schirmer, A.: Heuristische verfahren zur maschinenbelegungsplanung bei reihenfertigung. Zeitschrift fr Operations Research 19 (1975) 195-211

[9] Park, Y., Pegden, C., Enscore, E.: A survey and evaluation of static flowshop scheduling heuristics. International Journal of Production Research 22 (1984) $127-141$

[10] Dutta, S., Cunningham, A.: Sequencing two-machine flow-shops with finite intermediate storage. Management Science 21 (1975) 989-996

[11] Reddi, S.: Sequencing with finite intermediate storage. Management Science 23 (1976) 19-25

[12] Papadimitriou, C., Kanellakis, P.: Flowshop scheduling with limited temporary storage. Journal of the Association for Computing Machinery 27 (1980) 533-554

[13] Nowicki, E.: The permutation flow shop with buffers: A tabu search approach. European Journal of Operational Research 116 (1999) 205-219

[14] Smutnicki, C.: A two-machine permutation flow shop scheduling problem with buffers. OR Spektrum 20 (1998) 229-235

[15] Leisten, R.: Flowshop sequencing problems with limited buffer storage. Internation Journal of Production Research 28(11) (1990) 2085-2100

[16] Brucker, P., Heitmann, S., Hurink, J.: Flow-shop problems with intermediate buffers. OR Spektrum 25 (2003) 549-574

[17] Brucker, P., Heitmann, S., Hurink, J., Nieberg, T.: Job-shop scheduling with limited capacity buffers. OR Spektrum 28 (2006) 151-176

[18] Mascic, A., Pacciarelli, D.: Job-shop scheduling with blocking and no-wait constraints. European Journal of Operational Research 143 (2002) 498-517

[19] Jaffar, J., Lassez, J.: Constraint logic programming. Technical Report 74, Department of Computer Science, Monash University 74 (1986)

[20] S., R.: Probabilistic constraint logic programming. AIMS Arbeitspapiere des Instituts fr Maschinelle Sprachverarbeitung Lehrstuhl fr Theoretische Computerlinguistik, Universitt Stuttgart 5(1) (1999)

[21] Färber, G.: Sequencing in mixed model non-permutation flowshop production line using constrained buffers. PhD Thesis, Universitat Politècnica de Catalunya, Spain (2006)

[22] Holland, J.: Genetic algorithms and the optimal allocation of trials. SIAM J. Comput. 2(2) (1973) 88-105

[23] Holland, J.: Adaptation in natural and artificial systems. University of Michigan Press, Ann Arbor (1975) s

[24] Bolat, A., Al-Harkan, I., Al-Harbi, B.: Flow-shop scheduling for three serial stations with the last two duplicate. Computers \& Operations Research 32(3) (2005) 647-667

[25] Levitin, G., Rubinovitz, J., Shnits, B.: A genetic algorithm for robotic assembly line balancing. European Journal of Operational Res. 168 (2006) 811-825

[26] Wang, L., Zhang, L., Zheng, D.: An effective hybrid genetic algorithm for flow shop scheduling with limited buffers. Computers \& Operations Research (2006) Article in Press.

[27] Ruiz, R., Maroto, C.: A genetic algorithm for hybrid flowshops with sequence dependent setup times and machine eligibility. European Journal of Operational Research 169(3) (2006) 781-800

[28] Michaelewicz, Z.: Genetic Algorithms + Data Structures = Evolution Programs. 3rd edn. Springer Verlag (1996)

[29] Färber, G., Coves, A.: Performance study of a genetic algorithm for sequencing in mixed model non-permutation flowshops using constrained buffers. Springer Lecture Notes in Computer Science ISSN: 0302-9743, 3982/2006 (2006) 638-648 